\title{
Floating thrombus in the Ascending Aorta: Patient management and surgical treatment options
}

\author{
Martin Schmiady ${ }^{1}$, Thomas Horisberger ${ }^{2}$, Michael Hofmann ${ }^{1}$, Mathias van Hemelrijck ${ }^{1}$, \\ Francesco Maisano ${ }^{1}$, and Ahmed Ouda ${ }^{3}$ \\ ${ }^{1}$ UniversitatsSpital Zurich \\ ${ }^{2}$ UniversityHospital Zurich \\ ${ }^{3}$ University Heart Center Zurich
}

April 28, 2020

\begin{abstract}
A floating thrombus formation in the ascending aorta is a rare finding, which poses great challenges for treating physicians. Treatment strategies for this pathology are poorly defined and mainly depending on the clinical experience of the team. Using a case study, we discuss the underlying pathology, treatment options and patient management.
\end{abstract}

\section{Case description}

A 51-year-old male was referred to our center due to the incidental finding of a floating thrombus in the thoracic aorta. Two days before he suffered a transient ischemic attack with dysphasia and reduced vigilance. Due to an unclear focus of infection, a CT scan was performed, revealing a floating thrombus formation in the ascending aorta (Figure A and B). Due to two ischemic events in the past, we indicated the need for urgent thrombectomy to avoid further thromboembolic complications. Intraoperative transesophageal echocardiography confirmed the Diagnose of a pedunculated mass, originating from the posterior wall of the ascending aorta (Figure C and D). Open surgical thrombectomy through median sternotomy on cardiopulmonary bypass enabled the retrieval of the $11 \times 10 \mathrm{~mm}$ thrombus without fragmentation (Figure 1E). The attachement point showed a small endothelian leasion, caused by a ruptured vasa vasorum or sclerotic plaque. To prevent thrombotic recurrence, the site was covered by a pericardial patch plastic (Figure F). Postoperative course was uneventful. Histological examination revealed that the mass was a thrombus. The patient was discharged home under oral anticoagulation with phenprocoumon 7 days after intervention. Tumor screening and extensive coagulation diagnostics yielded no further findings, so that the anticoagulation could be stopped after three months. No recurrence or concomitant vascular embolism was observed during 6 month follow up.

\section{Discussion}

A mural thrombus of the thoracic aorta, that develops in the absence of pre-existing aortic disease, is a rare clinical finding with potentially devastating complications. Thrombus formation in the ascending aorta is an even more unusual event reported hardly before in patients with a non-aneurysmatic aorta ${ }^{12}$. The etiology of thrombus formation in a macroscopically normal aorta is not well understood. A correlation with underlying malignant disease, hypercoagulable disorders, endothelian disorders, steroid therapy, blunt trauma or even iatrogenic causes has been suggested ${ }^{345}$. In the presented case routine laboratory tests showed no abnormal findings and a coagulation disorder was excluded postoperative. Screening for cancer remains negative and the patient didn't had any B-symptoms. In the presented case, an endothelial lesion caused by a plaque rupture or the rupture of a vasa vasorum seems to be the most obvious origin of the thrombus formation. 
As this remains a rare disease, there is no consensus on prognostic assessments or optimal treatment strategy. Therapeutic options include therapeutic anticoagulation, open surgical thrombectomy and endovascular treatment with stentgrafts. Medical treatment (anticoagulation, thrombolysis) may lead to complete dissolution of the thrombus in most of the cases. However, a careful patient selection is necessary and embolization remains the major concern with this approach ${ }^{6}$. Open surgery with segmental aortic resection provides optimal treatment in patients with local neoplasms or endothelial lesions of the thoracic aorta and eliminates the risk of embolic events. In the case of endothelial damage, the affected segment should be replaced, or if localized, like in our case, a patch repair should be used to cover the thrombogenic spot. Cardiovascular bypass should be established by cannulation of the subclavian or alternative femoral artery, in order to prevent cerebral thromboembolism. Preparation of the aorta before cross clamping is performed in notouch technique. The endovascular approach is a much less invasive option than open surgery. However, the anatomical conditions must be suitable for stent release and there is an ongoing risk of distal embolism during intervention and wire manipulation.

Patient management includes a detailed patient history, clarification of coagulation disorders and tumor screening. A peripheral pulse status and possibly a Doppler sonography are part of the initial examination. Aortic CTA is recommended as first-choise examination because of availability and high sensitivity. For further evaluation of thrombus morphology, and to exclude intracardiac thrombi, a transthoracic or transesophageal echocardiography should be performed. A cranial CT is recommended in case of neurological symptoms. The therapy strategy is based on the thrombus morphology, location, patient age/ concomitant diseases and patient's wishes. In case of a mobile, pedunculated mass with localization in the ascending aorta open surgical thrombectomy should be considered, due to high thromboembolic risk. The indication for surgery should be made particularly generous in young patients. In case of thrombus localization in the descending aorta endovascular stent graft exclusion seems to be an effective and safe option. It can be considered as well for failed medical and open surgical approach. Mural, broad based thrombi, behind the outlet of the left subclavian artery may be suitable for initial medical therapy, especially in high risk patients.

\section{Conclusion}

The present case is intended to sensitize treating physicians for this uncommon disease. Even if it is a rare source for thromboembolic events, it must be considered in the differential diagnosis of embolic events. Cardiovascular surgeons should be aware of this pathology and the possible therapeutic approaches. The treatment of such complex cases requires the expertise of various specialists in the fields of cardiology, radiology, cardiovascular surgery and hematology, so that treatment in large centers is recommendable.

\section{References}

1. Wang B, Ma D, Cao D, Man X. Huge thrombus in the ascending aorta: a case report and literature review. J Cardiothorac Surg . 2019;14(1):157. doi:10.1186/s13019-019-0975-y.

2. Tsilimparis N, Hanack U, Pisimisis G, Yousefi S, Wintzer C, Rückert RI. Thrombus in the Non-aneurysmal, Non-atherosclerotic Descending Thoracic Aorta - An Unusual Source of Arterial Embolism. Eur J Vasc Endovasc Surg . 2011;41(4):450-457. doi:10.1016/j.ejvs.2010.11.004.

3. Onwuanyi A, Sachdeva R, Hamirani K, Islam M, Parris R. Multiple Aortic Thrombi Associated With Protein C and S Deficiency. Mayo Clin Proc . 2001;76(3):319-322. doi:10.4065/76.3.319.

4. Losanoff JE, Richman BW, Amiridze N, Rider KD, Jones JW. Floating thrombus of the thoracic aorta: a rare consequence of blunt trauma.J Trauma . 2004;57(4):892-894. http://www.ncbi.nlm.nih.gov/pubmed/15514551.

Accessed August 4, 2018.

5. Kloppenburg GTL, Sonker U, Schepens MAA. Intra-aortic balloon pump related thrombus in the proximal descending thoracic aorta with peripheral emboli. J Invasive Cardiol . 2009;21(6):e110-2. http://www.ncbi.nlm.nih.gov/pubme Accessed August 4, 2018. 
6. Choukroun EM, Labrousse LM, Madonna FP, Deville C. Mobile Thrombus of the Thoracic Aorta: Diagnosis and Treatment in 9 Cases. Ann Vasc Surg . 2002;16(6):714-722. doi:10.1007/S10016-001-0314-2.

\section{Compliance with ethical standards:}

All procedures performed in this case were in accordance with the ethical standards of the institutional and/or national research committee and with the 1964 Helsinki declaration and its later amendments or comparable ethical standards. Informed consent was obtained from the patient included in this work.

\section{Declaration of Conflicting Interests}

All authors declared no potential conflict of interest with respect to the research, authorship, and/or publication of this article.

\section{Funding}

None of the authors received financial support for research, authorship, and/or publication of this article.

Figure 1

Figure 1. Preoperative CT scan showing a floating, pedunculated mass in the ascending aorta (A and B). Perioperative transesophageal echocardiography confirmed the finding. The mass in located at the posterior wall of the aorta and floats in the bloodstream in a pulse-synchronized manner (C and D). Intraoperative photograph showing thrombus mass attached on the aorta (E). Patch repair after open thrombectomy (F).
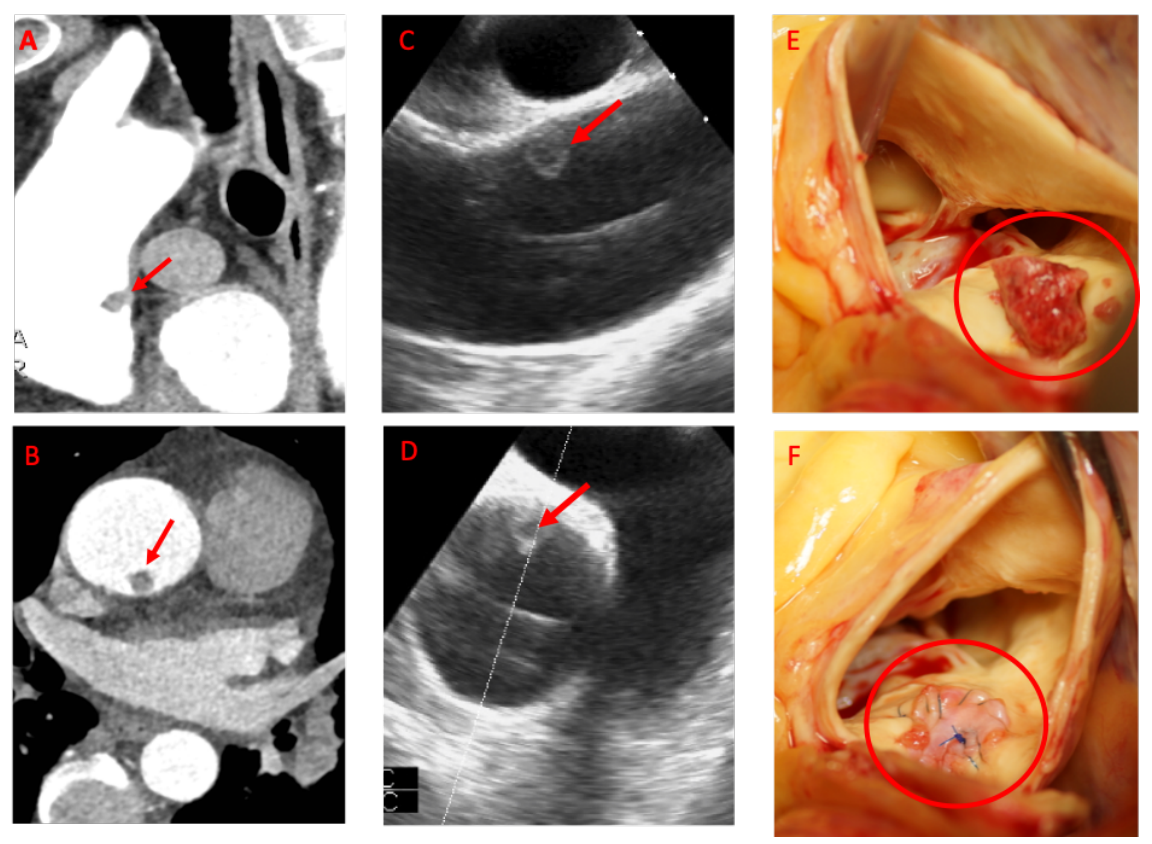